\title{
Collaborative Monocular SLAM with Multiple Micro Aerial Vehicles
}

\author{
Christian Forster ${ }^{1}$, Simon Lynen ${ }^{2}$, Laurent Kneip ${ }^{2}$, Davide Scaramuzza ${ }^{1}$
}

\begin{abstract}
This paper presents a framework for collaborative localization and mapping with multiple Micro Aerial Vehicles (MAVs) in unknown environments. Each MAV estimates its motion individually using an onboard, monocular visual odometry algorithm. The system of MAVs acts as a distributed preprocessor that streams only features of selected keyframes and relative-pose estimates to a centralized ground station. The ground station creates an individual map for each MAV and merges them together whenever it detects overlaps. This allows the MAVs to express their position in a common, global coordinate frame. The key to real-time performance is the design of data-structures and processes that allow multiple threads to concurrently read and modify the same map. The presented framework is tested in both indoor and outdoor environments with up to three MAVs. To the best of our knowledge, this is the first work on real-time collaborative monocular SLAM, which has also been applied to MAVs.
\end{abstract}

\section{INTRODUCTION}

\section{A. Motivation}

Micro aerial vehicles will soon play a major role in missions, such as security surveillance, search and rescue, and environment inspection. However, for such operations, navigating based on GPS information only is not sufficient. Fully autonomous operation in urban environments and indoor spaces requires micro helicopters to rely on alternative localization systems. However, weight restriction and battery autonomy impose great limitations on the choice of the sensors. For small-sized and lightweight platforms (less than $40 \mathrm{~cm}$ and less than $1 \mathrm{~kg}$ ), laser scanners are still too heavy and consume too much power. Therefore, the only viable solution is to use a combination of onboard cameras and IMU (Inertial Measurement Unit). Successful demonstrations of a MAV performing autonomous basic maneuvers, using only a single onboard camera, IMU, and an onboard Atom computer, have been done in our previous work [1], [2]. In this paper, we attempt to go one step forward, and address the problem of collaborative localization and mapping with multiple MAVs in unknown environments.

The application to multiple agents allows the use of redundant and parallel mechanisms to achieve increased robustness and efficiency. Several tasks- such as the workload of mapping an environment - can be shared among all the agents. As a practical result, the shared map among the

${ }^{1}$ C. Forster and D. Scaramuzza are with the Artificial Intelligence LabRobotics and Perception Group, University of Zurich, Switzerland-http: //rpg.ifi.uzh.ch

${ }^{2}$ S. Lynen and L. Kneip are with the Autonomous Systems Lab, ETH Zurich, Switzerland.

This research was supported by the Swiss National Science Foundation through project number 200021-143607 ("Swarm of Flying Cameras") and the National Centre of Competence in Research Robotics. robots allows the computation of the relative configuration of the agents, which forms a basis for multi-robot path planning and cooperative behaviors. Despite these advantages, solving the Simultaneous-Localization-And-Mapping $(S L A M)$ problem with multiple robots generally increases the computational and inter-robot communication load.

\section{B. Related Work}

Most works in multi-robot SLAM have been done using range sensors (e.g., laser, sonars, and stereovision) and/or ground mobile robots moving in the same 2D plane [3]-[6]. Very little work has been done using bearing-only sensors (monocular vision) and for unconstrained $(6 \mathrm{DoF})$ motion of the agents (e.g., wearable sensors, hand-held cameras, and flying robots). This problem-known as multi-camera structure from motion or multi-camera SLAM - can be approached differently depending on whether the cameras (i.e., the robots) can "see" each other or not. If the former case, their relative configuration can be inferred from the relative bearing-angle observations [7], [8]. In the latter case, this can be done starting from the common scene observed by the cameras. The work described in this paper belongs to the second category.

In [9], the authors use a single extended Kalman filter SLAM algorithm with an extended state vector composed of each camera pose and the observed features. Specifying the relative configuration at startup, they demonstrate results on two cameras attached to two bicycles. In [10], the authors describe a system for cooperative mapping using both aerial and ground robots equipped with stereo cameras. Each robot creates local submaps using an extended Kalman filter and maintains a global graph of submap positions. Rendezvous between robots, feature correspondences, and absolute GPS localization measurements, trigger loop closures which results in exchange of submap positions among the robots. In [11], the authors study the case of two MAVs which, equipped with monocular cameras and IMU, form a flexible stereo rig. Using feature correspondence in the overlapping field of view, the relative pose of the two robots can be estimated. In [12], the authors process the video streams from multiple hand-held cameras. The process is synchronized in that the images from all the cameras are processed all at once at each time step. This makes their system impractical for robotic applications, where the input of each camera should be computed asynchronously in order to cope with missing data and delays. Additionally, it is assumed that all cameras observe the same scene at start. In [13], a system was presented, where a single robot has to continuously localize within maps created during previous mapping sessions by 
the same robot. Although this work was not applied to multiple robots, it can, however, be seen as an instance of a multi-robot mapping process where each map was created in previous sessions by the same robot. Finally, in [14], a fully decentralized SLAM system is presented where each robot maintains a consistent augmented local map that combines local and neighbourhood information. The system has been validated in simulation.

\section{Contributions and Outline}

In the endeavor of enabling multi-robot navigation of MAVs with very-low onboard computing power, our goal is to employ the MAV onboard computer for low-level taskssuch as feature extraction, relative-motion estimation, and flight control—and delegate a ground station to higher-level tasks - such as mapping, loop-closure detection and map merging. The decoupling of motion estimation and mapping is useful in real-world scenarios, where the robots have to maintain some degree of autonomy in case of intermittent communication with the ground station.

An overview of the proposed approach is depicted in Figure 1. Each MAV estimates its motion individually by running an onboard visual odometry (VO) algorithm that is used to both track the robot motion and stabilize its $6 \mathrm{DoF}$ pose during flight. The outputs of the $\mathrm{VO}$-i.e. keyframe features and relative-pose estimates between keyframes-are streamed to a central ground station where our Collaborative Structure from Motion (CSfM) system is running. The CSfM system on the ground station creates an individual map for each MAV and merges them together whenever it detects overlaps. The ground station processes the data asynchronously, as it arrives, which accounts for situations where the robots do not start all at the same time or where some data are missing due to a communication failure. To achieve real-time performance, we design data-structures and processes that allow multiple threads (one for each MAV) to concurrently read and modify the same map. Additionally, we devise a solution to tackle the scale-difference between the onboard-estimated trajectories and those estimated on the ground station.

The remainder of the paper is structured as follows. Section III provides an overview of the CSfM system. Section III details the general mapping pipeline. Section IV] explains how overlaps between maps are detected and how they are merged into a single global map. Section $\mathrm{V}$ describes the implementation design for concurrent map access. Finally, Section $\mathrm{VI}$ provides the experimental results.

\section{SYSTEM OVERVIEW}

Each MAV tracks its own position using a keyframebased onboard monocular VO algorithm. We chose to employ the VO presented in our previous work [15]. It is boosted in terms of robustness and efficiency through the use of the relative-rotation prior from the onboard IMU. However, our proposed CSfM system is modular and, therefore, any alternative keyframe-based VO algorithm (such as [16]) could be used.

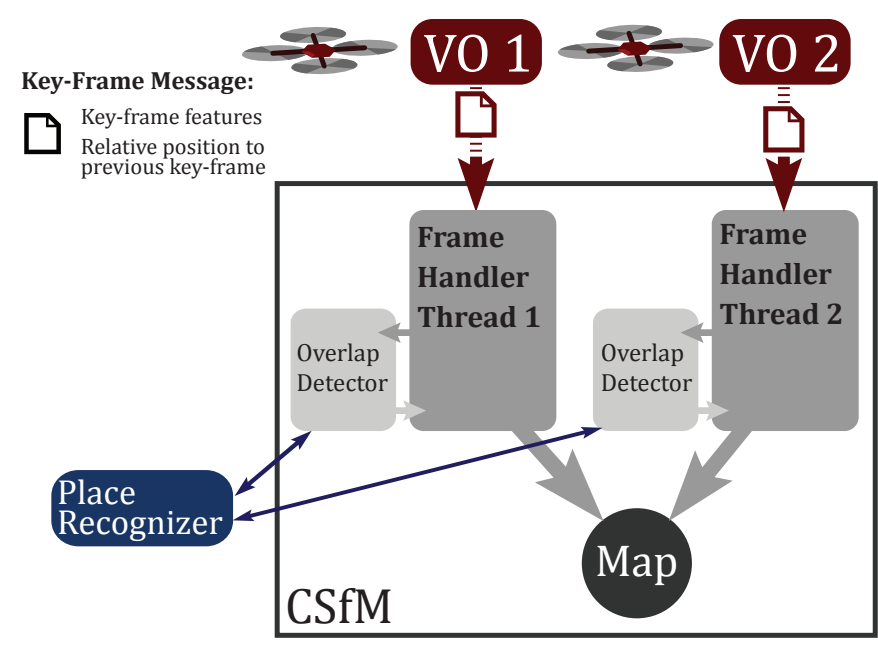

Fig. 1: The CSfM system-running on the ground station-creates a separate thread for each MAV. Initially, each thread creates its own map. However, the maps are merged when the Place Recognizer detects an overlap between the two maps. Both threads then read and update the same map simultaneously.

Figure 11 illustrates how the CSfM system is embedded in the multi-robot mapping framework. For each MAV, the CSfM system (running on the ground station) creates a new frame-handler thread that receives directly the keyframe messages from the corresponding VO. The frame handler creates a new map for its MAV and processes the received keyframe messages in parallel and asynchronously to the other frame-handler threads. A keyframe message only contains the extracted image features (i.e., image coordinates and descriptors) along with a relative transformation to the previous keyframe.

A keyframe is only added to a map when it provides new information. Right after a frame handler decides to add a keyframe to the map, it passes it on to its own overlapdetection thread (see Figure 22. The overlap detectors in turn pass the keyframes on to a place-recognition module. The place recognizer accumulates the visual information (i.e., feature descriptors), from all keyframes in every map, and quickly detects whether a place has been visited before. Meanwhile, the frame handlers triangulate new points and perform local Bundle Adjustment (BA) in the current keyframe's neighborhood. If the overlap detector detects an overlap within the map of the same MAV, a loop-closure optimization is initiated. Conversely, if the overlap occurs with the map of another MAV, the affected frame handlers are temporarily suspended to allow merging of the maps into a single one.

After map merging, the frame handlers operate on the merged map. Specially-designed data structures and the use of $\mathrm{C}++$ concurrency-control mechanisms allow multiple frame-handler threads to safely access and update the common map, which is also the key to real-time performance.

\section{MAPPING PIPELINE}

Figure 2 illustrates the mapping pipeline as implemented in the frame handler. The following sections detail the individual building blocks. 


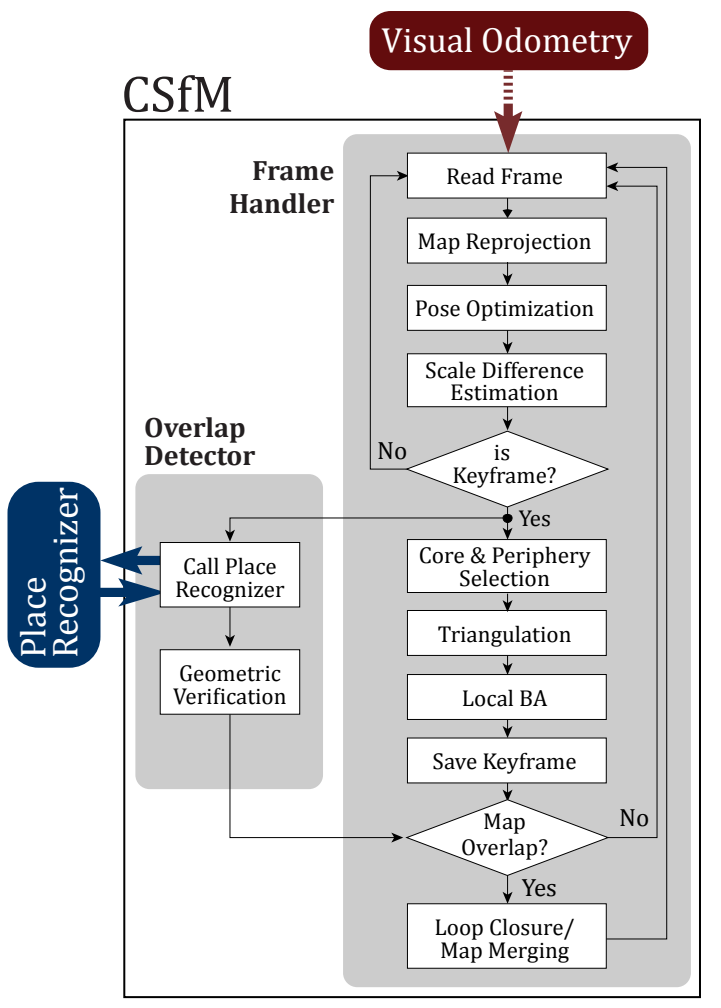

Fig. 2: Mapping pipeline executed inside the frame-handling threads of the CSfM system.

\section{A. Keyframe Message}

Each MAV tracks its own position (with respect to its own starting point) using a keyframe-based onboard monocular VO algorithm. When the onboard VO selects a new keyframe, a message to the ground station is sent containing the extracted features along with the relative transformation $\left(\hat{\mathbf{R}}_{k-1, k}, \hat{\mathbf{t}}_{k-1, k}\right)$ to the previous keyframe.

\section{B. Handling the Keyframe Message by the Ground Station}

When the ground station receives a keyframe message from a MAV, there are two possibilities: (i) if this is the first message from that MAV, then the CSfM system (running on the ground station) creates a new frame-handler thread and triangulates the received features into map-points as soon as the next message arrives; (ii) if a frame-handler for that MAV already exists, correspondences between the existing 3D map-points and the features in the new keyframe are identified. Additionally, the frame-handler updates the absolute pose $\left(\mathbf{t}_{k}, \mathbf{R}_{k}\right)$ of the new keyframe in the map:

$$
\begin{aligned}
\mathbf{t}_{k} & =\mathbf{t}_{k-1}+\mathbf{R}_{k-1} \hat{\mathbf{t}}_{k-1, k} \\
\mathbf{R}_{k} & =\mathbf{R}_{k-1} \hat{\mathbf{R}}_{k-1, k} .
\end{aligned}
$$

\section{Pose Optimization}

The CSfM system optimizes the absolute $6 \mathrm{DoF}$ pose of the new keyframe within the map by minimizing the reprojection error of all map-points visible by that keyframe using a nonlinear least-squares solver [17] 1

\footnotetext{
${ }^{1}$ The reprojection error is the Euclidean distance $e$ between the reprojected point and the corresponding observed feature in the image plane.
}

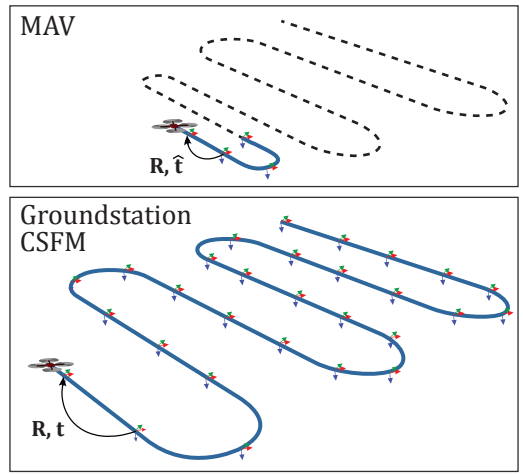

Fig. 3: The VO on the MAV maintains a map with a limited number of keyframes (e.g., 5) for processing-speed reasons. Therefore, the scale of the onboard VO drifts faster than on the ground-station. The relative translation $\hat{t}$ computed by the MAV's onboard VO needs to be corrected with the scale factor $\lambda$ for the CSfM map.

\section{Scale-Difference Estimation between VO and CSfM}

Each MAV's onboard monocular VO produces motion and structure information only up to an unknown scale factor. Furthermore, this scale factor is not constant, but drifts over time. On the ground-station side, the CSfM system also exhibits a scale drift as long as no loop closures occur. These two scale factors are not equal and diverge at different rates (see Figure 3). If the scale difference is not corrected, scale jumps can occur as it is depicted in Figure 6 (refer to Section VI. A scale jump occurs if the MAV's VO's scale drifts too much with respect to the CSfM map such that in the reprojection step no correspondences can be found and thus the pose cannot be optimized anymore towards the right position.

To correct this scale difference, we compare the estimated relative translation $\mathbf{t}$ before and after the pose optimization step:

$$
\hat{\lambda}_{k}=\frac{\left\|\mathbf{t}_{k-1, k \text { after Optimization }}\right\|}{\left\|\hat{\mathbf{t}}_{k-1, k \text { before Optimization } \|}\right\|}
$$

Hence, we compute the new scale factor $\lambda_{k}$ with the following update rule:

$$
\lambda_{k}=\lambda_{k-1}+\kappa \cdot\left(\hat{\lambda}_{k}-\lambda_{k-1}\right),
$$

where $\kappa$ represents the smoothing factor. Empirically, we found that $\kappa=0.05$ is a good choice.

Using the estimated scale-difference factor $\lambda_{k}$, the relative position received from the MAV's onboard VO is corrected by the corresponding frame-handler before a new keyframe is used. The position computed by Equation (1) is then updated to:

$$
\mathbf{t}_{k}=\mathbf{t}_{k-1}+\lambda_{k} \mathbf{R}_{k-1} \hat{\mathbf{t}}_{k-1, k} .
$$

This step further justifies why the pose of keyframes that are later not inserted in the map must also be optimized. It allows us to successfully track the robot's pose with respect to the map and to estimate the scale difference. 


\section{E. Keyframe Selection}

While the use of more map-points improves the accuracy of the map, increasing the number of keyframes has only minor effects once robustness is achieved [18]. Therefore, similar to [16], new keyframes are only inserted in the map if the distance to the closest keyframe is large enough ${ }^{2}$ Depending on the trajectory, the CSfM system rejects on average up to $85 \%$ of the received keyframes, which saves processing time.

\section{F. Selection of Core and Periphery Keyframes}

The CSfM system follows the fundamental concept that no temporal ordering of keyframes is retained. Keyframe neighbourhoods for optimization and triangulation are selected based only on spatial adjacency. This means that also older keyframes-regardless of the MAV they originate from-are taken into account for these operations, leading to a reduction of redundant information inside the map. A set $\mathcal{C}$ of four core keyframes is selected, which shares the largest number of common map-point observations with the new keyframe. The set of periphery keyframes $\mathcal{P}$ is defined by all keyframes that share at least one common map-point observation with $\mathcal{C}$ or with the new keyframe but which are not in the set $\mathcal{C}$.

\section{G. Triangulation}

New map-points are triangulated when a new keyframe is selected to be inserted in the map. For every unmatched feature in the new keyframe, we search matching features along the epipolar lines in the core keyframes. If a matching descriptor is found, the point is triangulated and projected into the remaining core and periphery keyframes to increase the number of measurements. The creation of duplicate points is inhibited by merging points in case a feature is already associated with an existing map-point. The merging step is essential for obtaining sparse and well constrained maps.

\section{H. Local Bundle Adjustment}

Mouragnon et al. [19] have shown the feasibility of creating an accurate $3 \mathrm{D}$ reconstruction in real-time using incremental bundle adjustment. Therefore, the CSfM system optimizes the set of core keyframes $\mathcal{C}$ together with the new keyframe and along with the commonly observed mappoints using the $g^{2} o$ framwork [17]. The set of periphery keyframes $\mathcal{P}$ is added to the optimization window with a fixed pose. The periphery keyframes are required to fix the scale of the structure and to ensure that the optimization is optimal with respect to the boundary.

\section{MAP OVERLAP DETECTION AND PROCESSING}

A fundamental characteristic of the CSfM system is its ability to detect if a MAV reenters an environment that has already been visited, either by itself or by another MAV which results in a loop-closure optimization or a

\footnotetext{
${ }^{2}$ We set the threshold to $15 \%$ of the average scene depth.
}

map merging respectively. Such overlaps are detected based on the keyframe appearance (i.e., feature descriptors) and subsequently geometrically verified.

\section{A. Appearance-based Overlap Detection}

If a keyframe is accepted for inclusion in the map, a second overlap-detection thread is started, which calls the place-recognizer module (see Figure 22. The external place recognition module is the same for all frame handlers and relies on a bag-of-words [20] approach. The exact type of place recognizer in use depends on the employed local invariant point descriptor. We initally tested OpenSURF features [21], which allow the use of the OpenFABMAP place recognizer [22]. However, for increased speed, we decided to use BRISK features [23]. Since binary features have special clustering properties, a dedicated place-recognition module was implemented $I^{3}$

\section{B. Geometric Verification}

Each time the place recognizer returns an overlapkeyframe with similar appearance as the current keyframe, the overlap detector geometrically verifies the result by applying the Perspective-Three-Point (P3P) algorithm from our previous work [24]. The P3P algorithm derives the camera pose from at least three 3D-to-2D feature correspondences. These correspondences are established by identifying matching descriptors between map-points-which the overlapkeyframe observes - and features in the current keyframe. To remove outliers, we integrated the P3P into a RANSAC [25] procedure. The output of RANSAC is then the rigid body transformation between the two keyframes.

\section{Map merging}

If the detected overlap occurred between two different maps, the similarity transformation $\{\mathbf{R}, \mathbf{t}, s\}$ returned by the geometric verification step is used to merge the two maps into one. The factor $s$ accounts for the different scale between the two maps and can be found by comparing the relative distances between any combination of 3D map-points which are common between the two maps. All frame handlers working on either of the two maps are temporarily suspended, and the entire candidate map for which an overlap was detected is subjected to the determined similarity transformation. To improve the measurements of points and avoid redundant information in the map, all map-points from each overlapping map region are reprojected into the keyframes from the other map and corresponding map-points are merged. A last important detail consists of applying the scale factor $s$ to the scale difference factor (see Section III-D) of all frame handlers that were operating on the transformed map. This is necessary to ensure that the received relative position estimates from the VO are correctly scaled with respect to the map. The frame-handler threads are finally resumed, and now operate in parallel on the same map. At this stage, it is

\footnotetext{
${ }^{3}$ The BRISK-based place-recognizer goes beyond the scope of this paper and, therefore, it is not described here.
} 
important to design the algorithm and data structures such that concurrent data access is possible (see Section V).

Note that the CSfM node creates references between two maps only when a loop closure is detected. However, in practice, the two maps may still contain overlaps in other regions if the place-recognition or the geometric-verification steps failed to detect them earlier. However, the CSfM system is still able to detect and incorporate them in a later stage in case a MAV retraverses the same environment.

\section{Loop closure}

The computed similarity transformation parameters $\{\mathbf{R}, \mathbf{t}, s\}$ also incorporate the amount of drift that has been accumulated along the loop.

The standard solution to optimize both the full map and keyframes after loop closure is to run global BA. However, this approach is computationally demanding and may fail completely due to convergence into local minima. Therefore, we chose to split the optimization into two steps. In the first step, we marginalize out the map-points. This reduces the map representation to a pose-graph with edges of different strength between poses. Strasdat et al. [26] were the first to propose 7-DoF pose-graph optimization including the scale as a drift parameter, which leads to a substantial improvement in a monocular-SLAM context. The parametrization of this pose-graph relaxation is included in the $\mathrm{g}^{2} \mathrm{o}$ framework [17] and used by the CSfM system. After pose-graph optimization, the map-points are updated accordingly and global BA is run to further refine both mappoints and keyframe poses simultaneously.

\section{IMPLEMENTATION DESIGN FOR CONCURRENT MAP ACCESS}

If two or more maps have been merged, multiple framehandling threads concurrently read and modify a single map (as depicted in Figure 11. Processing keyframes in parallel on a multi-core processor is the key to real-time performance of the CSfM system. However, when multiple things happen at the same time, special measures need to be taken both on the data-structure and on the algorithm layout level.

The difficulty of shared memory between multiple threads comes from the consequences of modifying data. We can ensure the integrity of the shared data by using the concept of mutual exclusion locks. This concept defines that if a thread wants to access some data-object, it first needs to acquire the data-object's lock, which is only possible if no other thread has previously acquired the lock without releasing it.

The design of data-structures defines the scope for simultaneous data access. Figure 4 illustrates the map data structure of the CSfM system. The map consists of a list of keyframes (KF-List), whereas each keyframe (KF) holds a list of references to map-points (MP) that it observes. The mappoints in turn also have a list of references to keyframes which they are observed by. There are only locks on the keyframe list and on the individual keyframes. If a thread owns the lock of a keyframe, it is allowed to read all mappoints which the keyframe observes. Hence-in order to

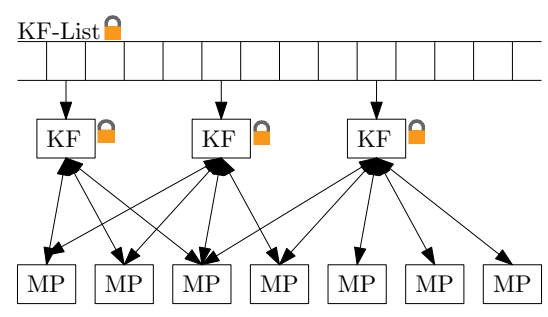

Fig. 4: Data-structure design for concurrency.

modify a map-point-it is necessary to acquire the locks of all keyframes that observe the map-point.

This property is used in the mapping pipeline: If a frame-handler thread locks all core and periphery keyframes $\{\mathcal{C}, \mathcal{P}\}$, it is allowed to modify these keyframes and all map-points which are observed by at least one of the corekeyframes $\mathcal{C}$. Fortunately, this is exactly the set of objects which change during local BA and triangulation.

Since no list of map-points exists, all map-points must be accessed via a keyframe. Knowledge of the lock state of this keyframe automatically inhibits that threads modify data that are out of their scope. Moreover, this design eliminates the overhead of locking individual map-points.

The employed locking strategy uses shared and upgradeable lock $\$^{4}$ which allows other threads to simultaneously read the data in the same neighborhood except for the negligible time when updates are saved in the map. If the MAVs operate in different parts of the map with non-overlapping core and periphery keyframes, they can even update the map concurrently.

\section{EXPERIMENTAL RESULTS}

Experiments were performed using two AscTec FireFly MAV $\$ 5$ equipped with an IMU, a single downlooking camera, and a Core-2-Duo computer. The ground-station was a $2.8 \mathrm{GHz}$ i7 laptop. A video of the experimental results is available at http://rpg.ifi.uzh.ch.

To evaluate its performance, our CSfM back-end was tested in both indoor and outdoor environments. Indoors, ground truth was obtained from a Vicon motion-capture system that provides absolute position information with millimeter accuracy at $100 \mathrm{~Hz}$. The output of the CSfM was evaluated by comparing the keyframe positions to the ground truth 6 The indoor environment consisted of a flat surface of approximately 8 by 8 meters. We added additional texture to the surface such that the VO algorithm has always enough features to track.

Figure 5 illustrates the influence of pose optimization (Section III-C) and local BA (Section III-H) on the median reprojection error of map-points. The peaks in the initial reprojection errors (blue dots) originate from the scale difference discussed in Section III-D As observed, they get

\footnotetext{
${ }^{4}$ Implemented e.g. in the Boost library: www. boost. org.

$5_{\text {www }}$.asctec.de/Firefly

${ }^{6}$ Since the scale of the map created by the CSfM system and the coordinate transformation between the computed and the ground truth trajectories are unknown, we derived the aligning similarity transformation $\{\overline{\mathbf{R}}, \overline{\mathbf{t}}, \bar{s}\}$ using a least-squares procedure.
} 


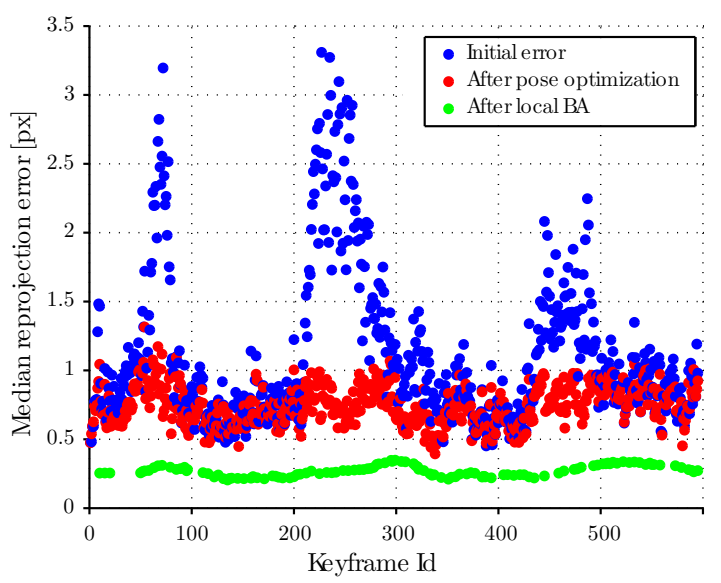

Fig. 5: Influence of pose optimization and local BA on the reprojection error in newly received keyframes.

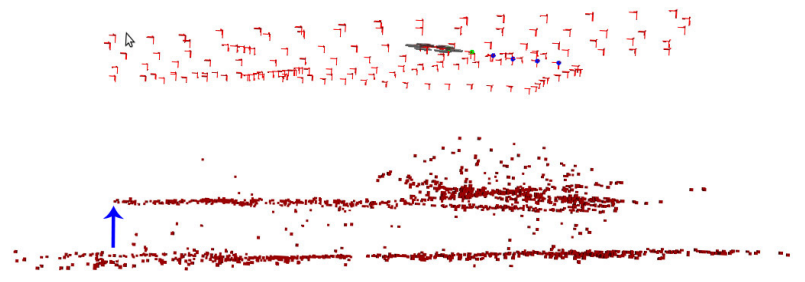

(a) Without scale correction. Scale jump is marked with a blue arrow.

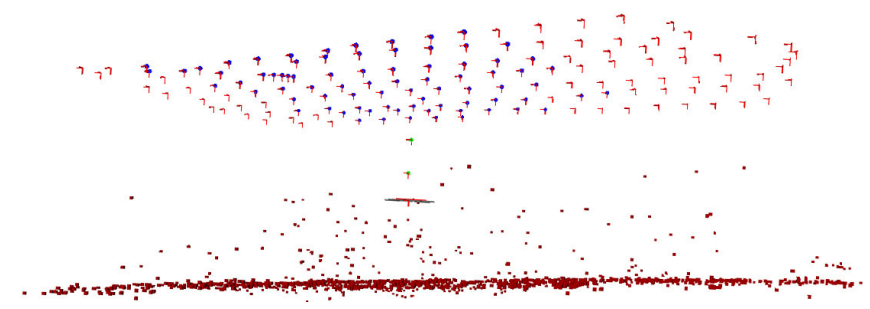

(b) With scale correction.

Fig. 6: Two maps of a flat surface seen from the side. The points on the bottom represent the map-points and the triangles on the top the key-frames. In (a), the scale jump of the map is clearly visible (blue arrow). In (b), the scale-difference estimation was activated and no scale-jump occurs.

canceled after pose optimization and local BA. Moreover, the peaks in the initial error disappear as soon as the scale difference factor $\lambda_{k}$ has adapted. Figure 6 shows the effect of scale difference estimation and correction that we mentioned in Section III-D If the scale drifts, the system is able to recover for reprojection errors up to 5 pixels. However, when the drift becomes too large, the system looses connection to the map and scale jumps occur. This effect does not arise if the scale is estimated and corrected, as shown in Figure 6p. The amount of drift depends on the chosen trajectory and on the distribution of the features in the keyframes.

Figure 7 shows a large loop trajectory before and after pose-graph optimization. On this trajectory, the system adopted 110 keyframes over $16.7 \mathrm{~m}$. The evolution of the error over time is reported in Figure 8 The loop closure occurs around $35 \mathrm{~s}$ and measurements are only indicated at times when a keyframe was created. The RMS error of the keyframe positions right before and after loop-closure detection and optimization was $0.1 \mathrm{~m}$ and $0.04 \mathrm{~m}$, respectively.

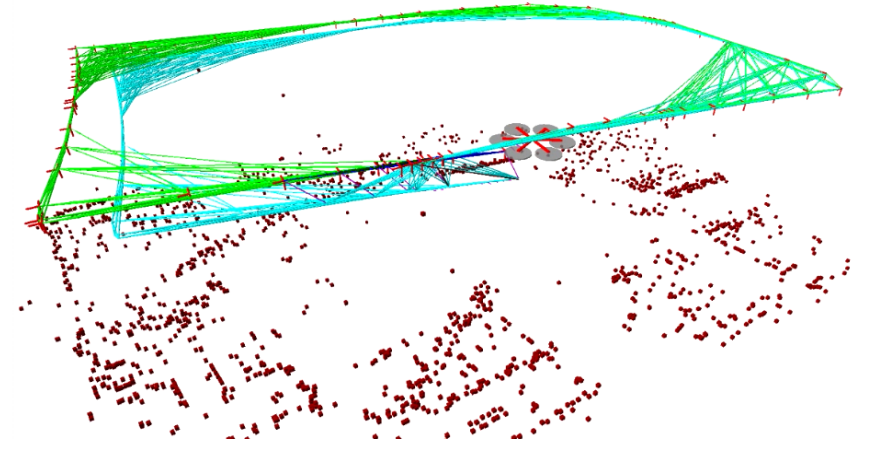

Fig. 7: 3D visualization of a pose-graph before (blue) and after (green) optimization on a loop trajectory.

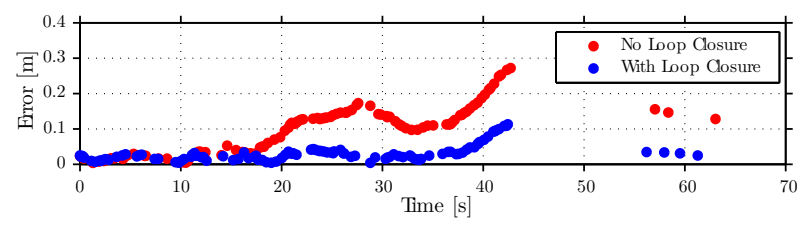

Fig. 8: Evolution of the RMS error of the loop trajectory in Figure 7 with and without explicit loop closure optimization. The loop closure occurs around $35 \mathrm{~s}$.

Figure 97 shows two MAVs simultaneously mapping two distinct areas. As soon as the CSfM system detects an overlap (b), it merges the two maps into a single, global one. Figure 10 shows comparison with ground truth obtained from a Vicon motion-capture system. Figure 11 indicates the corresponding error for both trajectories. The length of the combined trajectory was $30 \mathrm{~m}$, the total number of keyframes in the final map was 154, and the final RMS error $0.06 \mathrm{~m}$.

Our CSfM algorithm was also tested on two outdoor datasets from the European project sFly [27]. The combined trajectory length was approximately $400 \mathrm{~m}$ (see Figure 16 . Figures 12 to 15 show the mapping of the outdoor environment. Since both MAVs start at the same location, the two maps are immediately merged. Hence, the relative pose of the two MAVs is known from the beginning. Based on the color of the map-points, which is set to the color of the last MAV that observed it, one can see that both MAVs successfully localize in parts mapped by the other MAV (e.g., compare Figures 14 and 15. The GPS accuracy around the test area was ranging between 5 and 15 meters because of foliage and surrounding buildings. Therefore, GPS cannot be used as a reliable ground-truth. Nevertheless, some drift is still clearly visible between the estimated trajectory and the GPS. This is due to the absence of loop closures between the trajectories undergone by the two MAVs.

The most common failure case of the system occured when the place-recognition module missed to detect an overlap. In this case, after merging at a later stage, the global map contained redundant and, because of drift and map alignment errors, slightly misaligned map-points. The system was often able to recover from such situations through loop-closure detection and optimization at a second traversal.

By transmitting only binary features extracted from keyframes, the required bandwidth can be kept at a considerably low level ( $\sim 1 \mathrm{Mbit} / \mathrm{s}$ for 200 BRISK [23] features 
and $10 \mathrm{~Hz}$ keyframe rate) compared to streaming entire raw images $(\sim 86.6 \mathrm{Mbit} / \mathrm{s}$ for grayscale $752 \times 480$-pixel images and $30 \mathrm{~Hz}$ framerate). Note that the reduced keyframe-rate for our approach is because our VO already preselects a subset of frames as keyframes.

The average keyframe processing time on the groundstation ranged between $22 \mathrm{~ms}$ and $45 \mathrm{~ms}$, resulting in a frame rate of up to $45 \mathrm{~Hz}$ for one MAV. The average computation time per keyframe depends, to a large extend, on both the trajectory and the environment. A MAV that is constantly exploring new environments produces more keyframes-and, thus, a higher workload for the CSfM system - than a MAV that remains in previously-mapped areas. In the latter situation, most keyframes are dropped since they do not provide new information. On average the CSfM system selects only $85 \%$ of all received keyframes. Regarding the environment, an increased density of features implicitly leads to an increased number of map-point references and, thus, higher reprojection, matching and BA computation times. The average number or reprojected map-points ranged between 200 and 350. Furthermore, the efficiency of the algorithm also depends on inherent parameters, such as keyframe-selection criteria and size of the local BA window. The parallelized system pipeline is designed such that the processing time does not increase with higher numbers of MAVs_-given that for each MAV a processing core is provided. In experiments on the mentioned 4-core laptop, the processing time with two MAVs did not decrease significantly and with three MAVs real-time performance could still be achieved. One bottleneck is the place recognition module which currently is not parallelized and sequentializes the requests.

\section{CONCLUSION AND FUTURE WORK}

We proposed a system (named CSfM) for collaborative monocular SLAM with multiple MAVs using a centralized approach. By distributing the workload between the MAVs and the ground-station, we save processing power, require much less transmission bandwidth, and keep some autonomy on the MAVs themselves, i.e. the stability of the MAVs is not threatened by the reliability of the communication link. The CSfM system is highly modular and can work with different $\mathrm{VO}$ and place recognizer modules. We also presented a method for scale-difference correction, which solves an inherent problem of the decoupled system. The algorithm employs state-of-the-art techniques for active loop closure detection, bundle adjustment, and 7-DoF pose-graph relaxation. Results on real data including a comparison to ground truth demonstrate the high accuracy that can be achieved with vision-only SLAM. Finally, real-time performance was achieved with a system that allows multiple threads to concurrently read and modify the same map.

Future work will leverage on the potential to localize multiple MAVs in the same environment to allow purely vision-based coordinated flight of multiple robots.

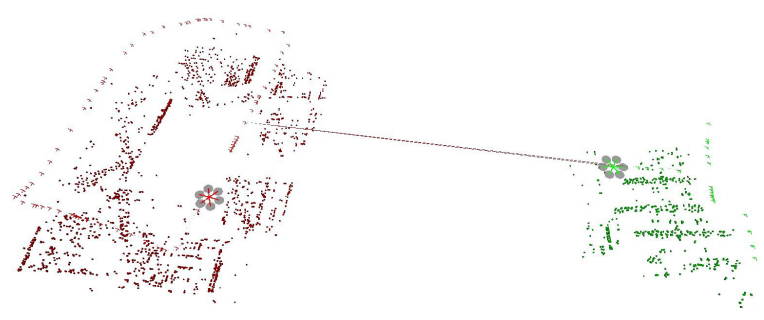

(a) before map merging

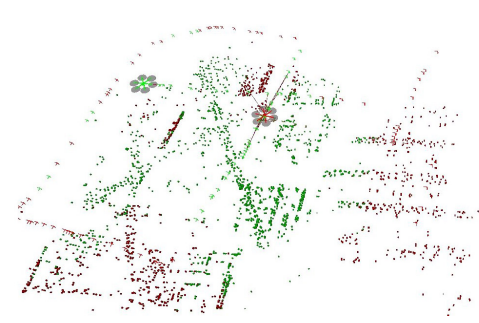

(b) after map merging

Fig. 9: Experimental results showing maps concurrently created by two MAVs in a real indoor environment. (a) The maps shortly after an overlap was detected by the place recognizer (red line). (b) The global map after merging.

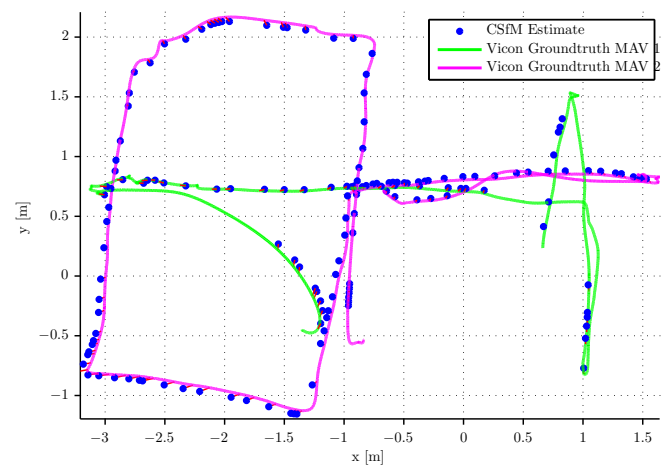

Fig. 10: The map of Figure 9p (after loop-closure and map-merging) is compared to the ground-truth. The blue dots mark the keyframe positions, while the green and purple lines are the ground-truth trajectories of both MAVs.

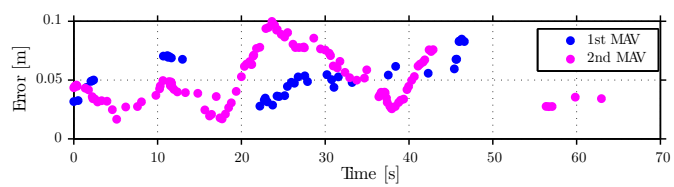

Fig. 11: Evolution of the RMS error of the keyframes in Figure 10

\section{REFERENCES}

[1] M. Blösch, S. Weiss, D. Scaramuzza, and R. Siegwart, "Vision based MAV navigation in unknown and unstructured environments," IEEE Proc. Int. Conf. on Robotics and Automation, 2010.

[2] S. Weiss, D. Scaramuzza, and R. Siegwart, "Monocular-SLAM-based navigation for autonomous micro helicopters in GPS-denied environments," Journal of Field Robotics, vol. 28, no. 6, pp. 854-874, 2011.

[3] D. Fox, W. Burgard, H. Kruppa, and S. Thrun, "A probabilistic approach to collaborative multi-robot localization," Autonomous Robots, 2000.

[4] R. Rocha, J. Dias, and A. Carvalho, "Cooperative multi-robot systems: A study of vision-based 3-d mapping using information theory," Journal of Robotics and Autonomous Systems, Vol 52, No 3-4, 2005.

[5] A. Howard, G. Sukhatme, and M. Mataric, "Multi-robot mapping using 

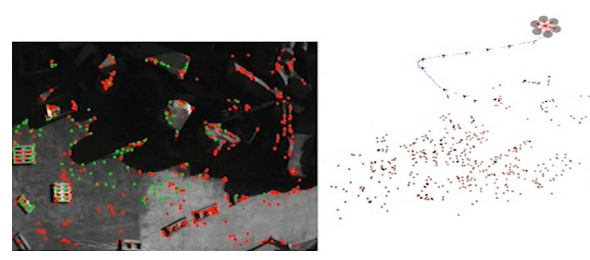

Fig. 12: The first MAV takes off and starts building its map.
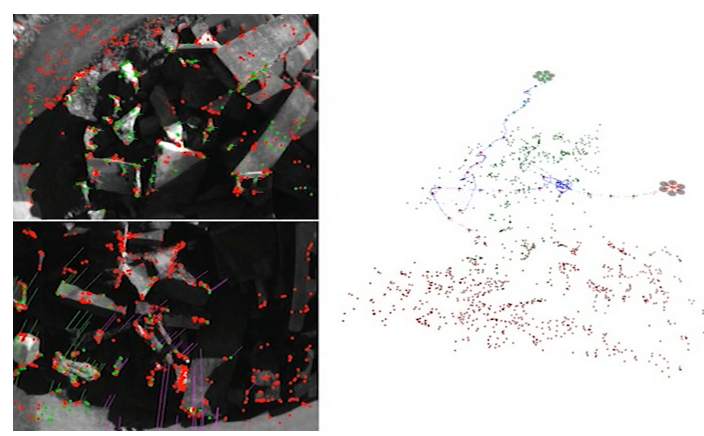

Fig. 13: The second MAV starts and immediately localizes in the map of the first MAV. The relative position of the two MAVs is now known.

manifold representations," Proceedings of the IEEE - Special Issue on Multi-robot Systems, 2006.

[6] N. Trawny, S. Roumeliotis, and G. Giannakis, "Cooperative multirobot localization under communication constraints," IEEE Proc. Int. Conf. on Robotics and Automation, 2009.

[7] A. Martinelli, F. Pont, and R. Siegwart, "Multi-robot localization using relative observations," IEEE Proc. Int. Conf. on Robotics and Automation, 2005 .

[8] M. Cognetti, P. Stegagno, A. Franchi, G. Oriolo, and H. H. Buelthoff, "3D mutual localization with anonymous bearing measurements," IEEE Proc. Int. Conf. on Robotics and Automation, 2012.

[9] J. Sola, A. Monin, M. Devy, and T. Vidal-Calleja, "Fusing monocular information in multicamera SLAM," Robotics: Science and Systems, 2008.

[10] T. a. Vidal-Calleja, C. Berger, J. Sola, and S. Lacroix, "Large scale multiple robot visual mapping with heterogeneous landmarks in semistructured terrain," Robotics and Autonomous Systems, 2011.

[11] M. Achtelik, S. Weiss, M. Chli, F. Dellaert, and R. Siegwart, "Collaborative Stereo," IEEE/RSJ International Conference on Intelligent Robots and Systems, 2011.

[12] Z. Danping and T. Ping, "CoSLAM: Collaborative visual slam in dynamic environments," IEEE Pattern Anal. Machine Intell., 2012.

[13] J. McDonald, M. Kaess, C. Cadena, J. Neira, and J. J. Leonard, "6-DOF Multi-session Visual SLAM using Anchor Nodes," Proc. European Conference on Mobile Robots, 2011.

[14] A. Cunningham, V. Indelman, and F. Dellaert, "DDF-SAM 2.0: Consistent distributed smoothing and mapping," IEEE Proc. Int. Conf. on Robotics and Automation, 2013.

[15] L. Kneip, M. Chli, and R. Siegwart, "Robust Real-Time Visual Odometry with a Single Camera and an IMU," Proc. British Machine Vision Conference, 2011.

[16] G. Klein and D. Murray, "Parallel Tracking and Mapping for Small AR Workspaces," IEEE and ACM International Symposium on Mixed and Augmented Reality, Nov. 2007.

[17] R. Kümmerle, G. Grisetti, and K. Konolige, "g2o: A General Framework for Graph Optimization," IEEE Proc. Int. Conf. on Robotics and Automation, 2011

[18] H. Strasdat, J. M. M. Montiel, and A. J. Davison, "Real-time Monocular SLAM: Why Filter?" IEEE Proc. Int. Conf. on Robotics and Automation, pp. $2657-2664,2010$.

[19] E. Mouragnon, M. Lhuillier, M. Dhome, F. Dekeyser, and P. Sayd, "3D Reconstruction of Complex Structures with Bundle Adjustment: an Incremental Approach," IEEE Proc. Int. Conf. on Robotics and Automation, 2006.

[20] J. Sivic and A. Zisserman, "Video Google: a text retrieval approach to object matching in videos," Int. Conf. on Computer Vision, 2003.

[21] C. Evans, Notes on the OpenSURF Library. University Bristol, 2009.
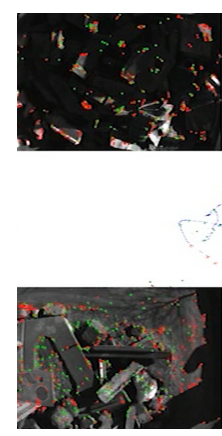

Fig. 14: The MAVs return to the take-off location.

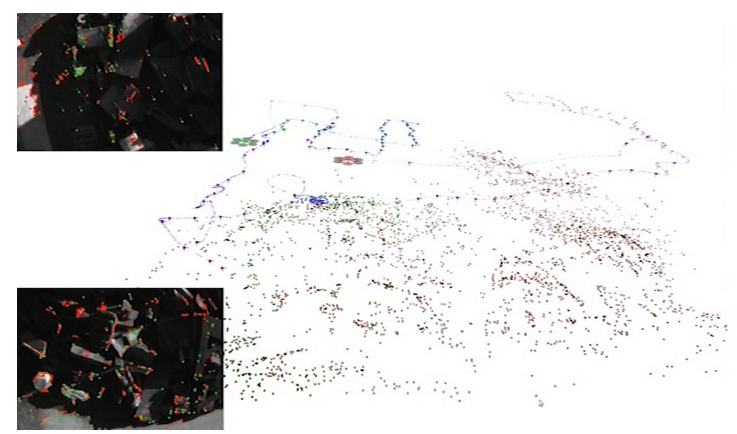

Fig. 15: Note that the color of the map-points indicate which MAV has last observed the points. One can observe that the red MAV observes and localizes with the map- points created by the green MAV.

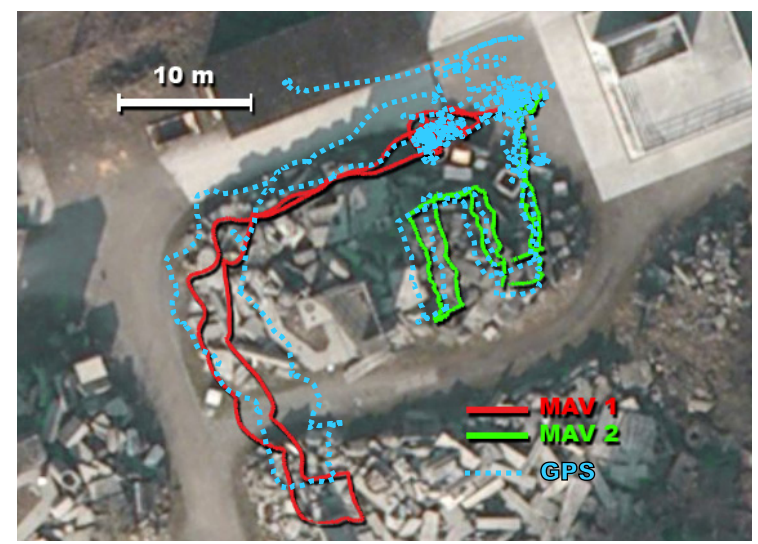

Fig. 16: Trajectories of the two MAVs in the outdoor experiment overlayed with the GPS measurements.

[22] A. J. Glover, W. P. Maddern, M. J. Milford, and G. F. Wyeth, "FABMAP + RatSLAM : appearance-based SLAM for multiple times of day," IEEE Proc. Int. Conf. on Robotics and Automation, 2010.

[23] S. Leutenegger, M. Chli, and R. Siegwart, "BRISK: Binary Robust Invariant Scalable Keypoints," Int. Conf. on Computer Vision, 2011.

[24] L. Kneip, D. Scaramuzza, and R. Siegwart, "A Novel Parametrization of the Perspective-Three-Point Problem for a Direct Computation of Absolute Camera Position and Orientation," IEEE Proc. Conf. on Computer Vision and Pattern Recognition, 2011.

[25] M. A. Fischler and R. C. Bolles, "Random Sample Consensus: A Paradigm for Model Fitting with Applications to Image Analysis and Automated Cartography," Communications of the ACM, 1981.

[26] H. Strasdat and A. J. Davison, "Scale Drift-Aware Large Scale Monocular SLAM," Robotics: Science and Systems, 2010.

[27] M. W. Achtelik, S. Lynen, S. Weiss, L. Kneip, M. Chli, and R. Siegwart, "Visual-Inertial SLAM for a Small Helicopter in Large Outdoor Environments," Video Proc. Int. Conf. on Intelligent Robots and Systems, 2012. 